The 5th International Symposium on Robotics in Construction June 6-8, 1988 Tokyo, Japan

\title{
21st CENTURY DEVELOPED BY ROBOTS
}

\author{
Ichiro Kato \\ Department of Mechanical Engineering \\ Waseda University \\ 3-4-1 Ookubo, Shinjuku-ku \\ Tokyo 160, Japan
}

\begin{abstract}
We have considered machines marvelous because they can do what man cannot do. We will have a new sense of value in the future, regarding machines as marvelous for doing things as well as man does.

In fact, it is easy for machines to do what man cannot do, and it is difficult for machines to imitate what man easily does. The difficult problems will be solved one after another in our age when technology is advancing at a rapid tempo.

Robots will then come out of the factories where they have been making products,' making their way far and wide into our living.
\end{abstract}

\section{Introduction}

Robots played an important role in the rapid growth of the secondary industries, and the tertiary industries gained a new dimension of importance along with it.

The growth of the secondary industries brought about expansion of distribution systems, growth of demand for consumer goods, and incidental expansion of the means of transportation and communication.

Such services as maintenance, cleaning, and repair grew as a new type of tertiary industry, and service industries associated with education and amusement were also expanded with the rising level of consumption and the increase of leisure.

Robots began to make their way into the expanding tertiary industries. The introduction of robots in this field is going to alter the sense of value that we have had. 


\section{Application to Services}

Apart from their application to the primary and secondary industries, robots began to be used in tertiary industries, for example, in the field of medical treatment. The technology of industrial robots for the primary and secondary industries is being directly applied to such areas of tertiary industries as transportation, warehousing, and cargo handling as well as maintenance and other services. An unattended system for supermarkets is one example. A maintenance robot for toll roads is another. The technology used for robots operating under extreme conditions can be directly applied to these robots. The technology for the so-called transport robot can be applied to the unattended transport of linen and meals in hospitals and hotels.

At any rate, such areas as transportation, warehousing, cargo handling, and maintenance - as in the construction industry - are behind others in automation so that they all share the problem of low productivity. It is for this reason that the introduction of robots into these areas is a matter of urgency.

There are signs of introduction of robots to restaurants for customer services and cooking.

There are several examples of application of robots to customer services, including one that takes orders and carries dishes in restaurants and another that substitutes company receptionists. Such robots are already in use for practical purposes.

Robots for educational and training purposes may also be mentioned if education is regarded as constituting one of the service industries. Apart from the dummy robot and simulator robot for medical treatment instruction and medical education mentioned, small robots for education in schools and universities and the robot called "Micromouse" that plays a labyrinth game may also be mentioned.

The robots to take part in the game must be fabricated by the participants themselves, making ingenious use of hardware and software. In this sense, these robots are not just for pleasure, but something that also produces significant educational effects.

\section{Amusement Robots}

The history of robots for popular entertainment dates back to the 18th century as can be witnessed from the three automata made by Jaquet-Droz and the automaton made by Vaucanson.

There are increasing instances of amusement robots displayed for attracting visitors and other purposes, using new technology.

Disneyland has been presenting a variety of shows and exhibits using a new type of mechanical human beings and animals developed on the basis of a technique called "animatronics" since about 20 years ago.

In Japan, a similar human robot of a highly expressive kind called Cybot (abbreviated from cybernetics robot) was developed by Mr. Toshikazu Mizuno. This robot is widely used in many exhibitions, performing theatrical acts with small movements of the hands and legs, and a variety of expressions with the eyes and mouth accompanied by music and words.

One of the largest events involving amusement robots was held in the Fuyo Pavilion, Tsukuba Expo. in 1985, where nearly 50 robots performed in a fantasic show. These robots embodying the existing high technology to the fullest possible extent performed as if they had intelligence in an impressive show involving communications with the companions. The show was indeed an occasion of giving a big dream to children. 


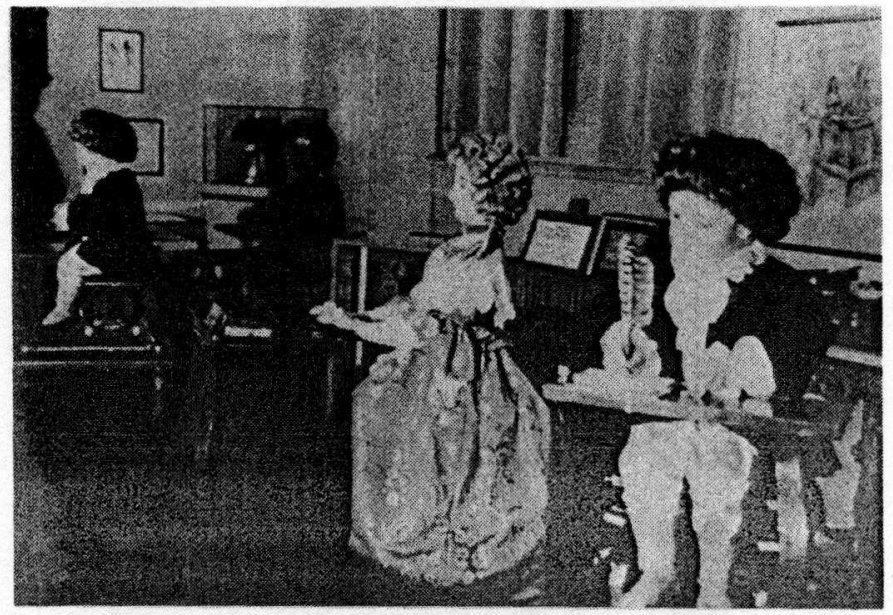

Fig. 1 Automata of Jaquet-Droz

(Historical Museum by the lake of Neuchatel, Switzerland)

There had been a considerably long period of automatic musical instruments in full bloom until Eidson invented the phonograph about a hundred years ago. They included devices that automatically played the piano, organ, violin, cello, or flute. There were so many of them developed and widely used, though they did not look like human beings.

The automatic music playing techniques show signs of recovery with recurrent popular preference to antique things. For example, automatic piano players were introduced to the market by some manufacturers, and seem to gain new acceptance. Present-day versions of violin and guitar playing machines have been made in several types in Japan. These fall in the category of automatic music player, and may not be called robots in a strict sense of the words.

\section{Musician Robot}

There must be many who still remember the musician robot shown in the Government Theme Pavilion, Tsukuba Expo. This was, and still is, an embodiment of the most advanced robot technology.

The musician robot can read musical scores that we human beings use, with an artificial eye, and play music directly from them. It takes only about 15 seconds to read a page of a musical score that the robot has not seen before, and determine the movements of its fingers. The robot then immediately starts playing. The robot has a far better finger function, in particular, than the human being. It hits the keyboard more than twice as fast as the human being with a single finger. Besides, the robot can talk with people in Japanese. It understands human language, and answers in Japanese in synthesized sound with a considerable degree of flexibility.

The robot can also play to a song in human voice. If some one finds the original key too high and sings in a lower key, the robot changes the key accordingly and plays to his song. All this indicates that the robot has a well advanced level of playing techniques, and has the capability of adjusting itself to the human being. 


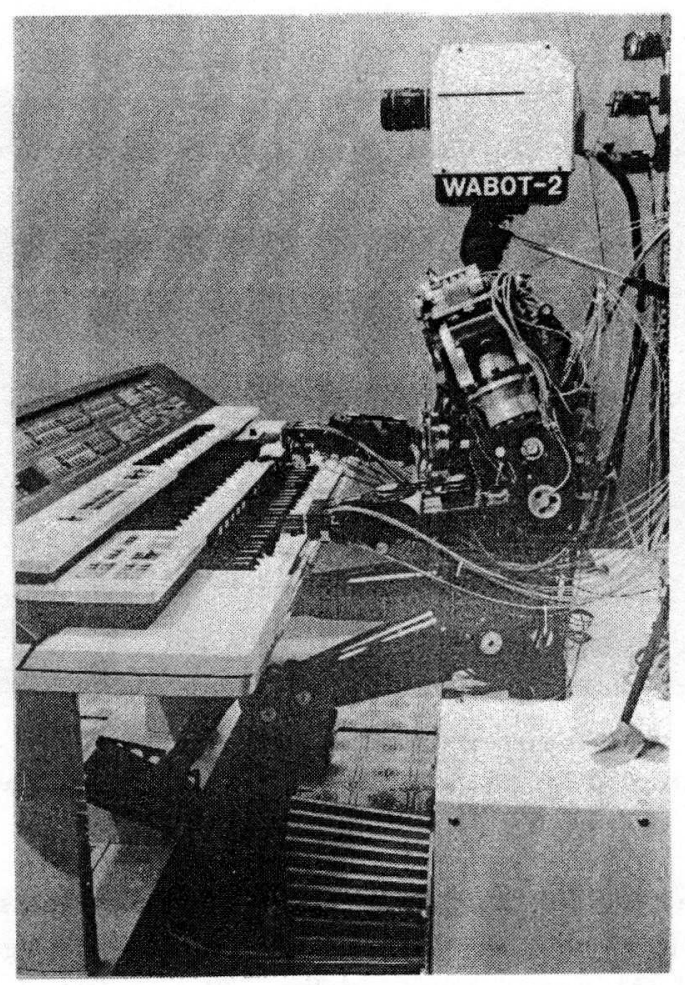

Fig. 2 WABOT-2

The robot has a degree of freedom of 21 for one hand and a degree of freedom of 4 for one leg, or a total degree of freedom of 50 for both hands and legs, with which the robot operates the organ as if it were a professional organ player.

The 50 joints must be controlled in a perfectly orderly way to fully use all this degree of freedom in exact accordance with a musical score and thus produce music. For this purpose, 78 microcomputers are arranged in series and parallel to constitute a triple hierarchical structure similar to that of the central control system of the human being to assure correct performance.

The computer-controlled hierarchical structure is similar to the triple structure of the human central control system consisting of the new cortex system, brain peripheral system, and brain stem spinal cord system.

Of the 78 microcomputers, those controlling vision, speech, and the motions of the hands and legs are arranged in parallel to constitute the lower level of control, those controlling lateral movements constitute the medium level of control, and those controlling the overall operation constitute the upper level of control.

The two types of capability that are incorporated into this robot - (1) dexterity and (2) adjusting itself to human beings - carry an important meaning in considering future robots.

The word "doll-like" is often used to express humorous mechanical movements. Present-day industrial robots operate doll-like, or machine-like. In contrast, human movements are smooth and pliant. The fact that this robot plays like a professional organ player means that it is now technically possible to achieve a significant level of flexibility in movement, which in turn allows dexterous movements. Another fact that the robot plays to the song sung by some one also carries an important meaning in considering the future relationship between man and robots or 
machines because it was human beings that had to adjust themselves to machines and other industrial robots.

\section{From Productivity to Amenity}

In other words, man will be able to live a life like man by turning robots human-like and using them in place of humans working like robots.

It might be added that industrial robots are making great contributions to the improvement of productivity, whereas robots in service areas are helping improve the amenity of life. This is indeed the ultimate goal of technology.

\section{Robots at Tsukuba Expo.}

You must still remember the Science Exposition held at Tsukuba in 1985. The two main themes were visual display and robots. Indeed, a great many robots were exhibited there. Those robots can be classified into three types as follows:

One is represented by the robot fantasy show presented in the Fuyo Pavilion. It was intended for children's entertainment, similar to the robot shows held in department stores to attract people. The robots in the fantasy show incorporated the latest technology to represent communications between man and robot. There, the robots acted as if they had intelligence.

Another is industrial robot. In the Tsukuba Expo., commercial robot hardware with a new type of software developed specially for the Expo. was exhibited in the respective pavilions. This type included one which wrote the names of persons on balls, another which drew portraits, and another which made ice sculptures.

Of those robots, the one which impressed me most was that which turned a top on the edge of a Japanese sword. One of the features of this show was the combination of a traditional Japanese art since the Edo period with high technology. It was a successful representation in the sense that the robot was highly accurate in positioning. The last type is a robot as a representation of high technology. The Government Theme Pavilion exhibited this type of robots. In the third section of the Government Pavilion called the "Human Corner", three robots were shown, including one that has four legs, another with two legs, and the last that plays the organ.

At any rate, the robots in the Tsukuba Expo. featured two specific points:

1. Intelligence

2. Information function

This suggests how robots will be toward the 21 st century.

\section{Meanings of WABOT-2}

People often ask why there was a four-legged robot in the "Human Corner" of the Government Theme Pavilion. Life originally used to swim like fish, which then evolved into a four-legged creature, which in turn evolved into a two-legged human being. As man walked on his two legs, the hands developed, thus enabling man to create civilization. One of the purposes was to show this process by means of a robot. 
Another purpose was to show how difficult it is to walk with two legs.

By overcoming this difficult problem, man developed the hands and created culture with the hands. One of the robots played the organ as an artistic performance, which is part of culture.

The fact that man walked with two legs had an important meaning because the hands developed from it, making man distinct from the other animals.

The organ-playing musician robot, WABOT-2, can do many things as mentioned before.

WABOT-2 was not originally intended to be shown at Tsukuba Expo. It was developed for two purposes. One was to create a dexterous robot, and the other was to build capabilities into it which would enable the robot to adapt itself to man.

Industrial robots are now used to do physical labor. Robots are machines likened to man. Man does not only physical labor but also information-related work, like writing and performing something artistic. If we want a robot to help us about things around us, apart from fabricating products in the factory, the robot must have two aspects like man. Therefore, this robot was designed to work dexterously and nimbly like the human being apart from doing physical labor. Now, about the capability of adjusting itself to man. All the machines that have been introduced since the industrial revolution, including industrial robots, do not adjust themselves to man. It is the human being that must adjust himself to them.

If a robot grows sophisticated to such an extent that it can help us about things around us, it must be easy to use by any one. Thus, it is the robot that must adjust itself to man, not vice versa.

WABOT-2 was an attempt to achieve this purpose, that is, to build into it the capability of adjusting the key and playing to the song sung by human beings.

\section{Age of Diverse Robots}

The Japan Industrial Robot Association calls 1980 the first year of robot. I personally regard the 1980's as the first phase of a robot age.

Industrial robots are now widely used. I would say that they are definitely a new type of automatic machines in terms of functions. They differ from the conventional automatic machines in that they have something like arms and memory devices to repeat the same thing. However, industrial robots are essentially the same as the conventional automatic machines.

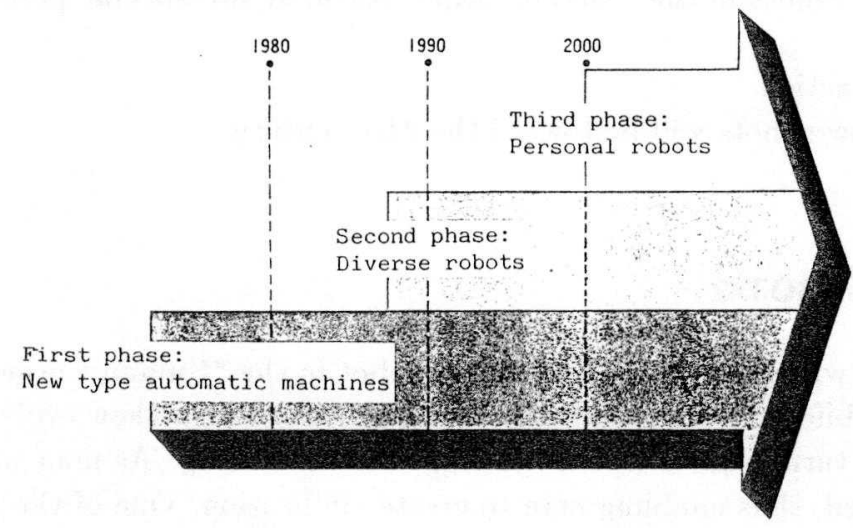

Fig. 3 New Era of Robot 
As mentioned before, "robot-like robots" are making their way. They may be considered in two phases: the second phase of the 1990's and the third phase that begins twenty years later in the 21st century.

The second phase of the robot age to come in about ten years is what I call a diverse robot age, in which a variety of robots will emerge. As you know, the industrial robots in use today are, in fact, single-purpose machines. Diverse robots to appear in ten years will also be single-purpose machines, but differ from the present counterparts in that they have advanced intelligence.

To have intelligence, sensors are necessary. The sensors now available are still a single-purpose type and large in size. New developments can be expected if a small-sized, integrated, multiple-function sensor is developed. But such a sensor is hardly likely in the next ten years. Therefore, I expect that a combination of various types of sensors with a computer will make a variety of robots having single-purpose intelligence. Such robots will be widely accepted for industrial purposes in the second phase of the robot age.

These diverse robots will be designed primarily for functions like the present industrial robots.

\section{Toward the Age of Personal Robots}

What will then be the next phase? I think that the third phase will come in the 21st century. I personally call it the personal robot age.

Apart from the present robots that produce things in the secondary industries, what I call personal robots will help us in the tertiary industries, in the fields of medicine and education, and at home to do things for us.

The robots used in the secondary industries play a role different from that played by the robots used in the tertiary industries. The users are also different. Personal robots will be used by people in general, not by specialists. Therefore, they must be easy to use.

Personal robots must have not only the dexterosity and the capability of adjusting itself to man that were mentioned before, but also diverse, multiple functions, not a single function, to do many things.

I expect that such robots will rapidly increase in the 21 st century for personal use, just like computers which were limited in number in the early days when they were large in size and used by specialists only. The emergence of microcomputers has changed the situation, raising predicted figures of computers to an estimated 50 million or even more in Japan alone.

Though not so much as computers, robots will also likely increase to 10 million or more for personal use.

\section{Symbiosis in Cybot Society}

Robot technology may also be regarded as intended for making artificial hands and legs as used by people who lost their hands or legs.

That is, robot technology can also be applied to the techniques of making artificial hands and legs. The techniques developed for robots are applied to electro-myogram controlled hands and legs.

A human being that uses a machine in place of a lost physical function is called a cyborg, or a 
cyborg man. With technological progress, there will be an increasing number of cyborgs in the 21 st century.

The next century will be an era of symbiosis between man, cyborg, and robot. Man learned to use tools in the primitive days. Tools and man have co-existed. Man invented machines through the industrial revolution, and learned to co-operate with machines. If man, cyborg, and robot are to live symbiotically in the 21 st century, the coming age may well be called an age of cybot society.

Robots, which are machines, are different from the conventional machines in that they have part B shown in the diagram in Fig. 4. In other words, robots will have a considerable part of the human functions. Part B will increase to expand the robot capabilities with technological progress, but my expectations are that man $\mathrm{A}$ and robot $\mathrm{C}$ will never be exactly the same, that is, robot $\mathrm{C}$ will never be man $\mathrm{A}$.

Thus, man will be the master in cybot society, and each of us, the human beings, must work toward creation of a flexible society in which man and machines will be able to live symbiotically in harmony.
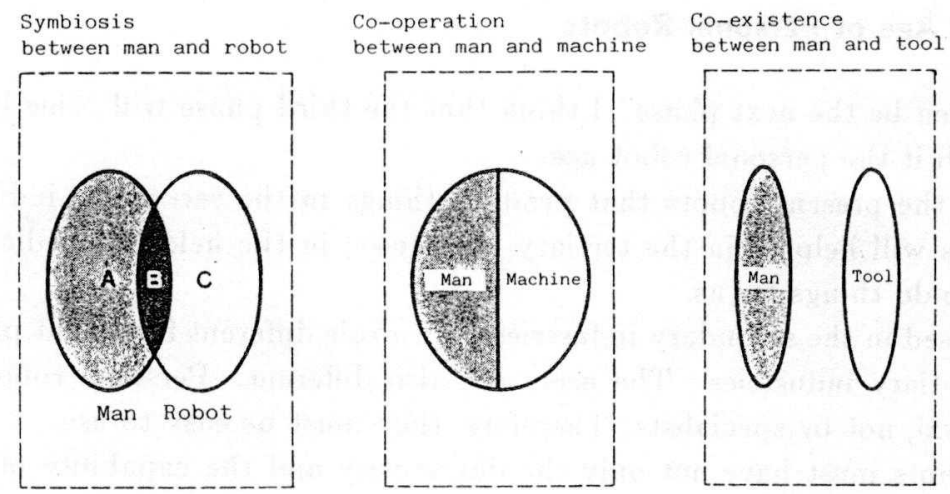

Fig. 4 Relations between Man, Tool and Machine 